\title{
La simulación como estrategia educativa para adquirir habilidades quirúrgicas
}

\author{
Simulation as an educational strategy to acquire surgical skills
}

\author{
Jesús Tapia-Jurado ${ }^{*}$ y Pamela Soltero-Rosas ${ }^{2}$ \\ ${ }^{1}$ Cirujano General, Presidente de la Academia Mexicana de Cirugía; ${ }^{2}$ Médico pasante en servicio social, Facultad de Medicina, UNAM. Ciudad de \\ México, México
}

La cirugía es una rama de la medicina que en los últimos 50 años ha tenido una evolución muy acelerada, llegando a ser menos traumática y mutilante. Sin embargo, por la aparición de nuevos procedimientos endoscópicos, el limitado equipamiento y el instrumental innovador, el aprendizaje de habilidades y destrezas quirúrgicas de mínima invasión no ha alcanzado la seguridad que requiere. El utilizar servicios médicos tiene un alto riesgo que condiciona una mortalidad de 1 por cada 1000 casos por año; en contraste, los servicios de aerolíneas comerciales suman 1 en cada millón. Por otro lado, se reporta que el error médico en el año 2016 fue la tercera causa de muerte, solo por debajo de los problemas de origen cardiaco y del cáncer1. Por tal motivo, hoy ya no es posible aprender exclusivamente frente al enfermo, sino que se está en la búsqueda de estrategias educativas que favorezcan un adecuado aprendizaje de la cirugía con el mínimo riesgo hacia los pacientes.

Según un estudio publicado por Mattar ${ }^{2}$, el $21 \%$ de los residentes no están preparados para operar, el $30 \%$ no son capaces de hacer por sí mismos una colecistectomía laparoscópica, el 24\% no identifican tempranamente signos de complicaciones, el $30 \%$ no son capaces de manipular tejidos en forma atraumática, el $26 \%$ no reconocen planos anatómicos y el $56 \%$ no realizan adecuadamente procedimientos de suturas. Por otro lado, empiezan a existir restricciones de horario de los residentes y tienen cada vez menos exposición a los enfermos y a los procedimientos quirúrgicos. Todo esto ha llevado a la búsqueda de estrategias educativas que permitan la adquisición de habilidades y destrezas quirúrgicas en modelos ajenos al paciente. Por tal motivo, ha surgido la simulación como estrategia educativa en medicina, la cual es una metodología de entrenamiento que reproduce la realidad en un ambiente controlado y cuya finalidad es adquirir y mejorar competencias profesionales, de comunicación, coordinación, liderazgo y, sobre todo, psicomotrices ${ }^{3}$.

La simulación para la adquisición de habilidades quirúrgicas tiene los siguientes objetivos: practicar todas las habilidades que demanda el currículum profesional, estandarizar desde un punto de vista docente los procedimientos quirúrgicos indispensables, repetir las prácticas cuantas veces sea necesario $y$, en consecuencia, aumentar la seguridad del paciente. Los beneficios de la simulación son: aprender en un entorno controlado, libre de riesgos y consecuencias adversas para el paciente; los errores que pueden suceder y que son inaceptables en la práctica clínica con el paciente, en la simulación se pueden permitir, analizando y discutiendo el error y ofreciendo la oportunidad para su corrección'; disminuye el tiempo de la curva de aprendizaje del alumno; y se optimiza y ahorra en recursos económicos, materiales y equipo de los quirófanos.

Es importante recordar que no estamos hablando de una estrategia nueva, ya que desde hace 2300 años Susruta fue el primero en utilizar la simulación, haciendo incisiones en un melón o con el uso de

\author{
Correspondencia: \\ *Jesús Tapia Jurado \\ Academia Mexicana de Cirugía \\ Av. Cuauhtémoc 330, 3o Bloque B \\ Unidad de Congresos del Centro Médico Nacional Siglo XXI \\ Col. Doctores \\ C.P. 06720, Ciudad de México, México \\ E-mail: tapiajj@amcg.org.mx \\ Fecha de recepción: 16-10-2017 \\ Fecha de aceptación: 13-12-2017 \\ DOI: $10.24875 /$ CIRU.M18000017
}

Cir Cir. 2018;86:5-7

Disponible en PubMed www.cirugiaycirujanos.com 
muñecas de lino para practicar vendajes. Sin embargo, ahora la simulación debe estar bien estandarizada y cubrir claramente los objetivos de aprendizaje. La simulación debe tener tres etapas fundamentales ${ }^{5}$ :

- Etapa cognitiva: en esta se conocerá cómo, para qué y por qué trabajar en el simulador y sus reglas educativas.

- Etapa integrativa: el alumno realiza las maniobras cuantas veces sea necesario hasta su dominio.

- Etapa autónoma: los movimientos manuales ya se realizan con destreza y en forma automática.

Podemos agregar una cuarta etapa, que es la evaluación, en la que se pretende demostrar que la habilidad practicada fue correctamente aprendida, para lo cual necesitamos sistemas de evaluación justos y objetivos.

Existen diferentes tipos de simulación. Tenemos la simulación no biológica, la cual se realiza en objetos inanimados de diferentes materiales, por ejemplo un brazo sintético en el que se realizan incisiones y suturas en repetidas ocasiones. Hay simuladores más complejos, con sistemas computarizados que les permiten manifestar diversos procesos patológicos e inclusive hablar. También hay simuladores virtuales, sobre todo para habilidades quirúrgicas de mínima invasión, en los que se realizan ejercicios básicos bimanuales y de profundidad ojo-mano, además de procedimientos quirúrgicos tipo colecistectomía, apendicectomía, histerectomía, prostatectomía, artroscopia de rodilla y hombro. E inclusive se dispone de simuladores para cirugía robótica. Los simuladores biológicos pueden ser de segmentos de animal inerte o híbridos (biológico y no biológico), de animal vivo tipo cerdo o de material humano inerte tipo cadáver; este último, gracias a las nuevas técnicas de preservación resulta un simulador de alta fidelidad, su entrenamiento ofrece realidad en la anatomía humana y la manipulación de tejidos, y además un mismo modelo inerte humano puede ser utilizado para diferentes procedimientos, pero tiene algunas desventajas, como son el costo, la ausencia de sangrado y la difícil disponibilidad 6 .

Han surgido en los últimos 10 años numerosos trabajos que afirman las cualidades del uso de la simulación. Por ejemplo, Draycott, et al. ${ }^{7}$ compararon en un estudio retrospectivo, observacional y comparativo, los problemas de distocia de hombro secundarios a una cesárea, antes y después del entrenamiento por simulación, demostrando que antes del curso el 17\% de los recién nacidos sufría tracción excesiva del plexo braquial y el $7 \%$ una lesión del mismo, mientras que en el grupo que recibió simulación solo el 9\% condicionaron tracción excesiva y disminuyó la lesión del plexo braquial al 2.3\%. En la cirugía laparoscópica se ha demostrado, comparativamente entre residentes que no hicieron simulación de ningún tipo y los que la recibieron mediante modelos virtuales, que estos adquirieron una mejor calidad de sus destrezas y habilidades quirúrgicas. También se ha demostrado que comparando los residentes que recibieron simulación con endotrainer contra los que recibieron simulación con realidad virtual, estos últimos tuvieron mejoras significativas en sus habilidades ${ }^{8}$. En un trabajo realizado por $\mathrm{Cox}^{9}$ se compararon residentes de tres grupos diferentes: sin ejercicios de simulación, con simulación virtual y con simulación en modelo animal. Los que realizaron práctica en modelo animal lograron un tiempo más corto en realizar una colecistectomía laparoscópica y su desempeño quirúrgico fue superior que el de los otros dos grupos, mejorando también la toma de decisiones, demostrando que el modelo de cerdo da fidelidad y existe disponibilidad del mismo; sus desventajas fueron el alto costo y las diferencias anatómicas. En neurocirugía ${ }^{10}$, usando cerdos de $40 \mathrm{~kg}$, el $97 \%$ de los residentes aprendieron a manejar complicaciones, mejoraron la disección del tejido cerebral y aprendieron el control de la hemostasia y del sangrado por succión, concluyendo que el modelo porcino vivo ofrece una adecuada experiencia para trabajar en un cerebro pulsante y con sangrado. En ortopedia11 se demostró que las simulaciones en modelos sintéticos mejoran la habilidad para la artroscopia, concluyendo que la simulación mejora el rendimiento quirúrgico en el paciente real. En la actualidad, el uso de modelos con impresora 3D ha evidenciado diferentes ventajas: el simulador es similar al paciente, se practica el acto quirúrgico cuantas veces sea necesario, permite al cirujano planear la cirugía y conocer las posibles complicaciones y cómo actuar ante ellas, e incluso evitarlas; pero como desventajas están la ausencia de sangrado, la poca disponibilidad de texturas con los materiales actuales y su costo elevado ${ }^{12}$.

Por lo que respecta a encontrar un sistema de evaluación más exacto y con el que podamos confirmar la adquisición de la habilidad enseñada, existen opciones, como listas de cotejo y evaluación por el experto. Con un sistema de dos cámaras y marcando los instrumentos que brindan un registro en computadora de los movimientos, asociado a análisis matemáticos, se compararon los movimientos entre alumnos de pregrado de la carrera de médico cirujano, residentes 
de cirugía general y cirujanos expertos con años de experiencia en cirugía laparoscópica, permitiendo la evaluación objetiva de los siguientes parámetros: destreza bimanual, longitud de trayectoria, percepción de profundidad, aceleración, energía del área y otros. Así, se logró ver la cantidad y la calidad de los movimientos de cada uno de los evaluados, con lo cual se tiene un parámetro más objetivo para evaluar la adquisición de habilidades quirúrgicas en laparoscopia ${ }^{13}$.

No es por demás decir que la simulación para la adquisición de habilidades y destrezas requiere una infraestructura compleja y costosa, la cual incluye diversas salas quirúrgicas, bioterio con personal certificado y capacitado para manipular los modelos biológicos vivos y un anfiteatro con procedimientos actuales para preservación de tejidos, los cuales deben cubrir la reglamentación que las instituciones certificadoras y sanitarias solicitan; por lo tanto, los grandes centros de simulación deben estar centralizados en las instituciones educativas de posgrado. Es importante mencionar que en dichos centros no solo se adquieren habilidades quirúrgicas, sino que a la vez se están conociendo los procedimientos quirúrgicos más actuales y el instrumental y el equipo más innovadores.

Finalmente, diremos que cualquier modelo de simulación sirve, independientemente de lo simple, sofisticado, barato o caro que sea, siempre y cuando tengamos presentes cuáles son los objetivos del aprendizaje. Además, se ha demostrado que la adquisición de habilidades y destrezas quirúrgicas es más rápida, que la educación se convierte en el único objetivo del ejercicio y que la simulación es una necesidad ética, ya que a los pacientes se les protege y no son objeto del aprendizaje.

\section{Bibliografía}

1. Makary M, Michael D. Medical error - the third leading cause of death in the US. BMJ. 2016;353:i2139.

2. Mattar SG. General surgery residency inadequately prepares trainees for fellowship: results of a survey of fellowship program directors. Ann Surg. 2013;258:440-9.

3. Tapia JJ. Los retos de la educación quirúrgica en el siglo XXI. Cir Gen. 2012;34:1-2.

4. Akhtar K, Chen A, Gupte S. The role of simulation in developing surgical skills. Curr Rev Musculoskelet Med. 2014;7:155-60.

5. Lazarini E, Schonstedt P, Abedrapo M, Yarmuch J, Csendes A, Rodríguez N. Simulación: una herramienta útil en la formación quirúrgica moderna. Rev Chilena de Cirugía. 2008;60:167-9.

6. Blackburn S, Griffin S. Role of simulation in training the next generation of endoscopist. World J Gastrointest Endosc. 2014;6:234-9.

7. Draycott T, Crofts J, Ash JP, Wilson LV, Yard E, Sibanda T, et al. Improving neonatal outcome tKhrough practical shoulder dystocia training. Obstet Gynecol. 2008;112:14-20.

8. Davis J, Khatib M, Bello F. Open surgical simulation- a review. J Surg Educ. 2013;70:618-27.

9. Cox M. Teaching surgical skills - changes in the wind. $N$ Engl $J$ Med. 2006;355:2664-9.

10. Regelsberger J, Eicker S, Siasios I, Hänggi D, Kirsch M, Horn P, et al. In vivo porcine training for cranial neurosurgery. Neurosurg Rev. 2015;38:157-63.

11. Akhtar K, Chen A, Gupte $S$. The role of simulation in developing surgical skills. Curr Rev Musculoskelet Med. 2014;7:155-60.

12. Waran VJ. Injecting realism in surgical training - initial simulation experience with custom 3D models. Surg Educ. 2014;71:193-7.

13. Pérez EF, Ordorica FRM, Oropesa GI, Zalles VC. Face, content, and construct validity of the EndoVis training system for objetive assessment of psychomotor skills of laparoscopic surgeons. Surg Endosc. 2015;29:3392-403. 